POST-TRANSLATIONAL MODIFICATION

\title{
Examining the Fic domain
}

The Fic domain, which is evolutionarily conserved and found in proteins from bacteria to humans, is the hallmark of a novel class of posttranslational modifying enzymes, Jack Dixon and colleagues report in Molecular Cell. These enzymes add AMP to Rho-family GTPases and can thereby mediate both bacterial pathogenesis and eukaryotic signalling.

Dixon and co-workers started their study by examining IbpA, a Fic domain-containing protein that is produced by the pathogenic bac-

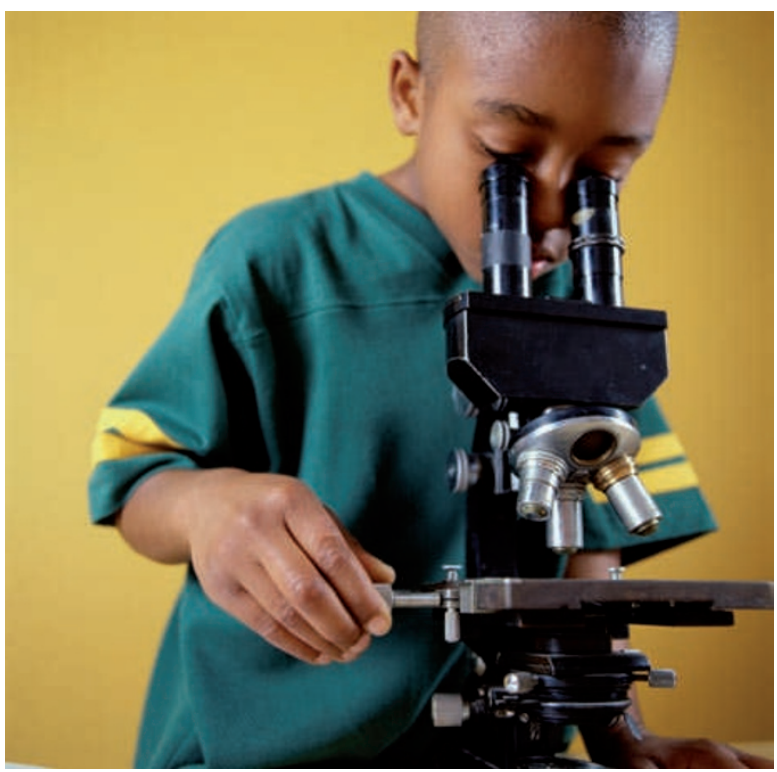

PHOTODISC terium Histophilus somni and has been implicated in H. somni virulence. Through analysis of a series of deletion mutations, they found that the Fic domain induces cytoskeletal collapse in HeLa cells, causing the cells to round up. Curiously, the effects of the Fic domain were reminiscent of the effects of proteins that target Rho-family GTPases. When the authors incubated a Fic domain with variants of RhoA, Rac and CDC42 and attempted pull-down assays, they found that there was no sustained direct interaction. Similar experiments showed that the Fic domain does, however, prevent Rho-family GTPases from interacting with their downstream effectors.

Perhaps, the authors thought, Fic functions by enzymatically modifying Rho-family GTPases. Analysis, by tandem mass spectrometry, of the products of in vitro adenylylation reactions involving non-radioactive ATP indicated that the Fic domain adds an AMP moiety to a conserved Tyr residue in the switch I region of RhoA, Rac and CDC42. This finding was supported by the results of in vitro adenylylation reactions that incorporated radiolabelled ATP. Moreover, treatment with a phosphodiesterase that can remove AMP from proteins indicated that the modification was reversible. The authors also found that the Fic domain targets active GTPases, rather than inactive GDP-bound GTPases.

Notably, these findings are relevant for $H$. somni pathogenesis - H. somni IbpA has the same function as purified Fic. Moreover, the Fic domain might also regulate key eukaryotic signalling events. HYPE, the only human protein that contains a Fic domain, also adds AMP to RhoA, Rac and CDC42, although it does not seem to inhibit the interaction of Rho-family GTPases with downstream effectors to the same extent as IbpA.

Taken together, the authors conclude that Fic domain-containing proteins are a novel class of enzymes that can both mediate bacterial pathogenesis and carry out a previously unrecognized eukaryotic post-translational modification.

Asher Mullard

ORIGINAL RESEARCH PAPER Worby, C. A. et al. The Fic domain: regulation of cell signaling by adenylylation. Mol. Cell 34, 93-103 (2009) FURTHER READING Yarbrough, M. L. et al. AMPylation of Rho GTPases by Vibrio VopS disrupts effector binding and downstream signaling. Science 323, 269-272 (2009) 